\section{Sostanza e forma nelle tecniche di procurement di farmaci aventi la medesima indicazione}

\section{Dear Editor,}

L'articolo di Andrea Messori et al "Le 'alternative terapeutiche': tecniche di procurement dei farmaci aventi la medesima indicazione terapeutica ma sprovvisti di parere di equivalenza rilasciato secondo la Legge Balduzzi", pubblicato su Global \& Regional Health Technology Assessment 2015; 2(3): DOI: 10.5301/GRHTA.5000202, merita di essere contestualizzato all'attuale normativa. Ricondurre a canoni giuridico-formali propri della normativa appalti la complessità e specificità dell'approvvigionamento dei farmaci nel cosiddetto canale ospedaliero è, di per sé, esercizio arduo. Anche perché il quadro regolatorio del farmaco, attraverso le sue norme speciali, incide direttamente sul relativo mercato. Basti pensare al regime dei prezzi. In questo contesto, la problematica dell'equivalenza terapeutica, da tradursi in comportamenti di acquisto, è esemplificativa delle discrasie anche concettuali che possono determinarsi tra acquisto del farmaco e regole formali di riferimento.

Una nota preliminare - se è consentita - riguarda il "sistema Balduzzi". A un osservatore esterno (quindi cui non siano note le pulsioni e le determinanti della "politica" del farmaco) appare quanto meno singolare che un'importante attività di HTA quale quella di confronto tra i principi attivi (produttiva di possibili rilevanti conseguenze sull'appropriatezza/contenimento della spesa sanitaria) venga lasciata al volontarismo delle Regioni e quasi subita dall'ente regolatorio che, sul tema, appare posizionato in stand by; quell'organismo che, appunto, dovrebbe presiedere con ruolo propulsivo alle politiche del farmaco, nell'interesse nazionale. Se, come è stato osservato, questo ente non viene dotato di adeguate risorse per tale attività, una ragione ci deve essere. Ovvio rilevare, poi, come il metodo dell'equivalenza on demand, nella sua farraginosità, si traduca in uno dei tanti casi di inefficienza generale di sistema e spreco di risorse (le stesse attività replicate in parallelo in ogni Regione, ritardi decisionali che comportano maggiori prezzi di acquisto ecc.).

La Regione Toscana attiva procedure ad interim, nella fase interlocutoria della pratica autorizzativa AIFA. In proposito, assumendo le categorie della normativa appalti ed esaminando le modalità di tali procedure, viene in campo il concetto di privativa o, meglio, di esclusività tecnica. Un determinato fabbisogno può essere soddisfatto solo da un determinato prodotto presente sul mercato e commercializzato in esclusiva, secondo una relazione biunivoca - relazione che sola giustifica la deroga alla gara pubblica. Sotto l'aspetto formale, delle due l'una: o il farmaco è, per determinati e preventivamente definiti utilizzi, in regime di esclusività tecnica ed è acquistabile da un unico operatore economico, e quindi ne è legittimo l'acquisto senza gara, o non lo è, e quindi per tali utilizzi va prevista la gara pubblica.

Per tali ragioni la prassi - richiamata nell'articolo in commento - di contrattualizzare tutti i farmaci appartenenti alle differenti classi terapeutiche, per poi orientare i consumi (in una sorta di equivalenza a valle dell'acquisto, di cui fu antesignana attuatrice, alcuni anni addietro, la Regione Marche) appare, sotto l'aspetto formale, di dubbia legittimità. Se tale sistema è qua e là operante è anche perché, non contrastando generalmente gli interessi del mercato, non trova opposizioni fatte valere in sede giurisdizionale. Non mancano tuttavia specifici pronunciamenti, come la sentenza del TAR Umbria n. 254 del 2013. Quel giudicato considerò illegittima la clausola di gara che prevedeva che "in seguito all'aggiudicazione la Commissione Terapeutica Regionale si riserva la facoltà di scelta delle molecole da utilizzare nelle strutture Regionali, nell'ambito di classi omogenee e sulla base di valutazioni farmaco-economiche complessive (prezzo offerto in gara e prezzo al pubblico)". In essa si affermava che "la pur riconosciuta specificità degli approvvigionamenti di farmaci e la flessibilità dello strumento prescelto del Sistema Dinamico di Acquisizione, non possono portare all'eccesso di consentire (...) una sorta di confronto concorrenziale postumo (...) non potendo comunque tali valutazioni di equivalenza trovare ingresso nella fase successiva all'aggiudicazione (...)".

Non coerenti con l'ordinamento appaiono anche offerte aperte, cioè che possono prevedere sconti/quantità, con riserva della stazione appaltante di determinare successivamente, in base all'entità degli sconti, le proprie convenienze economiche. Occorrerebbe individuare preventivamente ed esplicitare le quantità di confronto tra le offerte.

Una strada per centrare l'obiettivo dell'acquisto in maniera trasparente e, per quanto possibile, oggettiva, può essere quello della segmentazione della fornitura.

Potremmo quindi avere, nell'ambito della medesima categoria terapeutica, un acquisto separato per "profilo-paziente" (lotto/profilo singolo). Per il profilo A è idoneo solo il farmaco 1 (acquisto diretto), per il profilo B sono idonei i farmaci 1 e 2 (gara competitiva) ecc...II tutto fatte salve comunque ulteriori residuali eccezioni motivate. Si renderebbero tendenzialmente oggettive la larga parte delle scelte di acquisto. Una costruzione concettuale che, ovviamente, dovrebbe reggere prima di tutto sul piano scientifico. A superamento anche dell'abitudine prescrittiva o della continuità terapeutica nei casi non scientificamente giustificati.

Per inciso, l'ordinamento europeo sugli appalti enfatizza la definizione dell'oggetto del contratto in termini prestazionali, piuttosto che per contenuti merceologici, e ciò per meglio tutelare la concorrenza (nell'interesse di domanda e offerta). Un principio che sembrerebbe previsto apposta per favorire il confronto fra molecole diverse. In effetti, secondo l'ordinamento europeo: "Le specifiche tecniche devono consentire pari accesso agli offerenti e non devono comportare la creazione di ostacoli ingiustificati all'apertura dei contratti pubblici alla concorrenza. (...) Le specifiche tecniche sono formulate: (...) in termini di prestazioni o di requisiti funzionali (...) le stazioni appaltanti non possono respingere un'offerta di lavori, prodotti o servizi conformi (....) ad una specifica tecnica comune (...) se tali specifiche contemplano le prestazioni o i requisiti funzionali da esse prescritti (...). L'operatore economico che propone soluzioni equivalenti ai requisiti definiti dalle specifiche tecniche equivalenti lo segnala con separata dichiarazione che allega all'offerta" (1). 
Ne consegue che l'offerta di una molecola diversa da quella prevista dal bando con medesime indicazioni dovrebbe essere accettata, ovvero respinta, motivatamente.

Il punto rimane quindi quello della qualificazione di quel farmaco per quel dato utilizzo. La stringente connotazione dell'esclusività tecnica (e di ogni limitazione alla concorrenza con motivazioni tecniche), che dovrebbe avere base esclusivamente scientifica, mal si concilia con la soggettività di cui - anche in virtù della cultura medica individualista - è portatore il professionista clinico. Problematiche ben note, non affrontabili in questa sede, che, appunto, si cerca faticosamente di superare anche con l'attivazione degli studi di equivalenza. Il tutto, in un panorama nazionale di velocità diverse sui temi dell'appropriatezza e del controllo della spesa. Le modalità con cui vengono strutturate le procedure di acquisto dei farmaci nelle varie realtà esprimono il livello di approfondimento scientifico e/o di "volontà politica" di incidere sulla qualificazione della domanda, tanto che gli stessi prodotti finiscono per essere acquisiti con modalità e livelli di concorrenzialità diversi. A volte sulla base di compromessi scientifici o commerciali e norme disinvoltamente interpretate. Ciò che dovrebbe risultare, secondo l'ordinamento degli appalti pubblici, bianco o nero assume, nel caso del farmaco, svariate sfumature di grigio. Siamo appunto in presenza di una delle discrasie concettuali di cui si accennava in premessa.

La procedura posta in atto dalla Regione Toscana sembra prefigurare una qualificazione mista dell'acquisto. Si parte da una negoziazione diretta in privativa con più fornitori per poi, nella fase dell'esecuzione del contratto - cioè nella fase della dispensazione del farmaco per le diverse tipologie di pazienti - tenere conto dei risultati economici delle negoziazioni, in una sorta di valutazione concorrenziale ex-post che sembra prescindere, almeno in parte, dalla casistica clinica o dalla tipologia del paziente. Quella tipologia o casistica clinica che, negli atti di acquisto, doveva essere stata individuata a giustificazione dell'acquisto diretto di quel determinato farmaco. E questo, dal punto di vista della normativa appalti, potrebbe rappresentare una prima criticità. Sotto un profilo sostanziale, se a una data casistica clinica o tipologica di paziente possono essere associati farmaci diversi (scelti in funzione del prezzo), siamo di fronte a un'equivalenza terapeutica a valle della procedura di acquisto, pur non dichiarata. Verrebbe quindi meno il presupposto che ha legittimato l'acquisto diretto.

Si sarebbe quindi in presenza di una valutazione competitiva innestata su offerte in privativa, in contrasto con l'ordinamento comunitario che ha cassato, anche solo a proposito della possibilità di rinegoziare al ribasso il prezzo con l'aggiudicatario, l'ibridazione delle procedure (in quel caso una procedura negoziata innestata su gara pubblica) (2).

Ciò in coerenza formale con i principi della trasparenza e della par condicio, in base ai quali vanno esplicitati, negli atti propedeutici all'acquisto, i contenuti del contratto e i criteri (oggettivi e automatici) di scelta del contraente. Sono bandite dall'ordinamento scelte discrezionali o definite posteriormente all'esplicitazione delle offerte.

Non appare comunque illegittima un'aggiudicazione plurima (già attuata, ad esempio, in gare a lotto unico nazionale o pluriregionale, per evitare il formarsi di monopoli) (3) purché basata su una procedura pubblica e con criteri di aggiudicazione predefiniti. In sostanza, si tratta di prevedere una pro- cedura pubblica in cui è messa in gara l'indicazione terapeutica, sono predefinite le aliquote di aggiudicazione e i relativi criteri di attribuzione.

Sempre dal punto di vista normativo, assume rilievo la determinatezza dell'oggetto del contratto (costituito dalla tipologia del bene o servizio da fornire e dalla quantità prevista). È sancito in giurisprudenza che "anche le centrali di committenza hanno l'obbligo di attenersi sotto tutti i profili alle norme del codice dei contratti pubblici e quindi, al fine di dare attuazione ai principi di trasparenza e concorrenzialità, di rendere noto, nell'esperimento della procedura concorsuale, l'importo complessivo oggetto dell'appalto (...)" (4). Se la quantità non è preventivamente determinata, deve essere determinabile, cioè quanto meno devono essere predefiniti i criteri oggettivi di determinazione.

In conclusione, le osservazioni di natura giuridico-formale sulle procedure di acquisto potrebbero essere confinate nell'innocuo orticello dei dibattiti dottrinali su cui si appassionano i fini cultori del diritto, senonché le criticità formali che possono essere attribuite a procedure di acquisto non ortodosse rappresentano anche altrettante occasioni per l'instaurazione di contenziosi in sede giurisdizionale, con le immaginabili conseguenze sui costi e sull'efficienza degli acquisti. Uno spauracchio da rimuovere o, quanto meno, da minimizzare. Come? Derogando dall'ortodossia solo una tantum (secondo giurisprudenza, l'eccezione, in determinati casi, può legittimare la deroga) per ragioni di urgenza e superiore interesse pubblico, e potendo dimostrare al giudice di avere rispettato i principi nazionali e comunitari di legalità, trasparenza, par condicio.

\section{Disclosures}

Financial support: No financial support was received for this submission.

Conflict of interest: The author has no conflict of interest.

\section{Marco Boni}

Past-President, Federazione delle Associazioni Regionali Economi e Provveditori della Sanità (FARE), Verona

Indirizzo per la corrispondenza:

Marco Boni

Via Mercadante, 24

41122 Modena, Italy

marco.boni.15@alice.it

\section{Bibliografia}

1. Direttiva 2004/18/CE - art. 23; D. Lgs. n. 163/2006 - art. 68.

2. Circolare della Presidenza del Consiglio dei Ministri-Dipartimento per le politiche comunitarie, n. 12727 del 15 novembre 2001: "Divieto di rinegoziazione delle offerte nelle pubbliche gare dopo l'aggiudicazione".

3. Si veda, ad esempio: Consip - procedura ristretta SPC connettività (www.Consip.it).

4. TAR Toscana - sentenza n. 889/2015.

Published online: December 11, 2015 